\title{
Implementing a Mobile Campus Using MLE Moodle
}

\author{
Fatos Xhafa \\ Dept. of Languages and Informatics Systems \\ Technical University of Catalonia, Spain \\ EMail: fatos@lsi.upc.edu \\ Isaac Rustarazo \\ Dept. of Computer Science, Multimedia and Telecommunication \\ Open University of Catalonia, Spain \\ EMail: irustig@uoc.edu
}

\begin{abstract}
Mobile learning is considered the next step of online learning by incorporating mobility as a key requirement. Indeed, the current wide spread of mobile devices and wireless technologies brings an enormous potential to e-learning, in terms of ubiquity, pervasiveness, personalization, flexibility, and so on. For this reason, Mobile Learning is attracting significant research efforts covering a fairly variety of learning settings, from schools and universities to workplaces and cities. This research has evidenced that mobile technology can offer new opportunities for learners to learn inside and beyond the traditional instructor-oriented educational paradigm. However, mobile technologies are still in its infancy and many challenges arise. In this paper we analyze, from both learning and technological perspectives, the development of learning applications using mobile devices. To this end, proxy and proxyless architectures are considered as way to extend traditional virtual campuses with mobile clients. The objective is twofold: to access learning materials and to support learning activities. A prototype of a Virtual Campus is developed using MLE-Moodle -the Mobile LEarning module of Moodle. The proposed Virtual Campus enables mobile clients to perform online learning activities and is a step towards achieving the "anytime, anywhere" paradigm.
\end{abstract}

Keywords-Mobile Learning, Awareness, Mobile technologies, Pervasiveness, Web application, MLE Moodle.

\section{INTRODUCTION}

Over the last decade, we have witnessed an explosion of mobile devices and wireless technologies for communication and for sharing many types of informational resources. While this has dramatically transformed our society in the way we communicate, create, retrieve and share information, collaborate and socialize each other, the application of these technologies to certain sectors of society is still in its infancy [35], [36]. Education, in the form of electronic learning and teaching, is still far from making the most of mobile technologies to support the day-to-day classrooms and enhance learning experiences and processes, however, great research efforts are increasingly being made to incorporate mobility in this domain.

In developing on-line environments that support mobile

\author{
Santi Caballé \\ Dept. of Computer Science, Multimedia and Telecommunication \\ Open University of Catalonia, Spain \\ EMail: scaballe@uoc.edu \\ Leonard Barolli \\ Dept. of Information and Communication Engineering \\ Fukuoka Institute of Technology, Japan \\ EMail: barolli@fit.ac.jp
}

learning, several issues must be taken into account in order to ensure full support to the online learning students. One key issue is mobility in correspondence with the current mobility learners and the widespread of mobile devices and wireless technologies [36]. Indeed, the proliferation of mobile phones and other handheld devices has transformed mobile learning from a researcher-led endeavor to an everyday activity, whereby mobile personal tools help people learn everywhere through either formal training or informal support, collaboration and conversation [17]. As a result, by the addition of mobility and the support of mobile technologies, the Mobile Learning (M-Learning) has become a reality [45].

M-Learning bases the success of current and future learning applications on the capability of such applications to incorporate mobility to support the learning processes. For this reason, this issue has already attracted the attention of researchers, pedagogues and developers of applications from e-learning [3], [9], [11]. Current literature in m-learning is however rather short though intensively increasing over the last years. As a result, m-learning is still in its infancy and many challenges are to be addressed before being fully benefited from incorporating mobility to day-to-day collaborative learning.

Mobility is seen by researchers and pedagogues [8] as a new opportunity for education since it provides more chances for learners to personalize their learning process, enhance the social interactions, learn more effectively and more autonomously, and collaborate with other peers and teachers at anytime and from anywhere, inside and outside the formal collaborative learning context. Indeed, both the capabilities of mobile devices and their wide context of use contribute to their propensity to foster interaction and collaboration. Mobile devices can easily communicate with other devices of the same or similar type, enabling learners to share data, files and messages. They can also be connected from anywhere at anytime to a shared data network, further enhancing possibilities for communication. These devices are also typically used in a group setting, and so interactions 
and collaboration will tend to take place not just through the devices but also at and around them as well.

A great variety of challenges arise though when using mobile devices, ranging from technical - such as how to manage devices with very small screens and keywords, which do not facilitate easy access to text and impede input or annotation - to educational - such as how to coordinate small learning groups in the classroom. And yet mobile collaborative learning is about supporting learners for both formal and informal collaborative learning activities in which they are willing to participate, seamlessly, with a greatest success paradoxically occurring at the point where they do not recognize it as learning at all [10].

In this paper we analyze, from both learning and technological perspectives the development of on learning applications using mobile devices. A prototype of Mobile Virtual Campus implemented in MLE-Moodle is then proposed. We start by providing related work on projects and environments devoted to support m-learning (Section II) and analyzing the most important learning and technological perspectives in Section III for the development of on learning applications using mobile devices. Then, alternatives of integrating mobile devices into a server-based Virtual Campus are presented (Section IV). Next, we describe in Section $\mathrm{V}$ the most appropriate architecture to extend the Virtual campus with mobile clients which becoming a Mobile Campus. A prototype of a Mobile Campus is developed in Section VI by using MLE-Moodle -the Mobile LEarning module of Moodle. The proposed application enables mobile clients to perform online learning activities and is a step towards achieving the "anytime, anywhere" paradigm. We end the paper in Section VII with some conclusions.

\section{BACKGROUND}

Despite the novelty of incorporating mobile devices into on-line learning, many mobile learning approaches, initiatives and projects have appeared over the last years [21]; [12]. Some representative efforts are:

Mature multi-funded M-LEARNING.ORG international project [26] has as a primary target of m-learning the young adults who are not taking part in education or training, or are mobile, casual, temporary or self-employed, or in low income/skills employment with literacy/numeracy development needs. The learning involves a series of very small, quick learning experiences designed to help development of literacy and numeracy skills.

SMILE (Sussex Mobile Interactive Learning Environment), is a mobile learning initiative that gave flexibility to learners in creating their own learning experiences [20]. Students were loaned mobile devices which had PDA capabilities, mobile office tools and always-on Internet connection. Students were asked to explore opportunities for using mobile devices for collaboration and communication. In this project, there was little work done towards automating or enhancing the process of accessing content as much as it was directed towards assessing the potential of the devices in enhancing the learning experience.

The UNITE project (Unified e-Learning environment for the school) [44] set out from a state-of-the-art analysis of the existing educational platforms, mobile learning and eLearning pedagogical frameworks with the view to structuring an eLearning environment capable to meet the learning needs of the schools it addressed. The main output of the project was the UNITE eLearning environment, as well as a series of eLearning scenarios covering a great range of thematic axes, which could be customized to the needs of each learning audience.

AMULETS (Advanced Mobile and Ubiquitous Learning Environments for Teachers and Students) project at $\mathrm{Vxj}$ University explores how to design, implement and evaluate innovative educational scenarios combining outdoors and indoors activities supported by mobile and ubiquitous computing. AMULETS is based on the premise that the design of innovative mobile learning activities should be guided by collaborative learning scenarios in context in authentic settings. Educational scenarios were designed together with teachers to support the regular curriculum in a wide range of topics including geography, history and biology [37].

The EXPLOAR project [13] demonstrates an innovative approach that involves visitors of science museums and science centers in extended episodes of playful learning. The EXPLOAR approach considers informal education as opportunity to transcend from traditional museum visits to a "feel and interact" user experience, allowing for learning "anytime, anywhere", open to societal changes and at the same time feeling culturally conscious. A set of demonstrator learning scenarios are being implemented employing advanced and highly interactive visualization technologies in personalised ubiquitous learning paradigms.

The recently launched (2010) $\mathrm{m}$-Voice Learning project at the Open University of Catalonia (UOC) [28] is the creation of a platform equipped with a VoIP communication system between students and teachers that enables the realization of a subject, or a part of it, through mobile devices and is compatible with the UOC Learning Management System. The main goals of the project are to create case studies that allow interaction, discussion and decision making in teams; adapt material presentation systems to facilitate discussion; and provide individual and group evaluation.

However, the technical and institutional problems faced by existing m-learning initiatives has led many researchers [21] to suggest that an integration with existing Virtual Learning Environments (VLEs) used by institutions can be beneficial. Approaches extending VLEs to m-learning are the e-learning lab in Shanghai [40] or the Finesse client [24]. Claroline is another VLE that was adapted for mobile access as a collaboration project between a mobile media company and the national college of Ireland [15]. This work resulted in 
stressing several technical issues that relates to designing engaging content for mobile learners and investigating approaches for adapting content for several mobile platforms. Finally, Engage [32] is another interesting project that aims to enable access of personalised learning material on mobile devices from several VLEs such as Domino, Moodle and uPortal.

Throughout this paper, a further example of integrating mobile access with the VLE of Moodle is introduced. MLearning is a specific module of Moodle platform [25]. By using this module it is possible to enable the online learning through MLOs (Mobile Learning Objects). MLOs are developed in Mobile Learning Engine Markup Language (MLE-ML) [27], which is the language to create contents to be consumed by a MLE client. The content can be stored in the mobile device and to which the user can have access to at any time. Contents created via MLE-ML range from simple text to audio and video.

\section{Paradigms and Technological Perspective FOR MOBILE LEARNING}

In this section, we consider many of the current pedagogical paradigms that support learning and give some clues to help understand the potential of using mobile devices in this context [8].

\section{A. Constructivist learning}

Constructivist's central idea is that human learning is constructed rather than received, that learners build new knowledge upon the foundation of previous learning. In order to transform learners from passive recipients of information to active constructors of knowledge instructors must give learners an environment in which to participate in the learning process, and the appropriate tools to work with that knowledge [6].

Mobile devices provide a unique opportunity to have learners embedded in a realistic environment at the same time as having access to supporting tools. Each learner carries a networked device which allows them to become part of the dynamic system they are learning about. Therefore, learners are able to experiment and learn from the environment by themselves.

\section{B. Behaviorist learning}

In this paradigm, learning is thought to be best facilitated through the reinforcement of an association between a particular stimulus and a response. The use of mobile devices can enhance the behaviorist learning process by stimulating students with the presentation of engaging teaching materials and tasks, obtaining responses from learners, and providing appropriate feedback (reinforcement). In addition, video games, which are replacing some curricula's teaching methods, are implemented using advanced portable video game consoles. They are used to compete in matches' against computer-generated opponents offering faster increasing process of stimuli-response-feedback [30].

\section{Situated Learning}

The situated learning paradigm [19] emphasizes the idea of cognitive apprenticeship where teachers (the experts) work alongside students (the apprentices) to create situations where the students can begin to work on problems even before they fully understand them. To this end, one important aspect in online learning systems is to provide learners with awareness, that is, knowledge about what other participants are doing at present and what they did in the past [14]. Situated learning requires knowledge to be presented in authentic contexts (settings and applications that would normally involve that knowledge) and learners to participate within a community of practice.

Mobile devices, such as PDA and smart phones, can support students in different learning contexts as well as reinforce the learning personalization and adaptation by the natural alliance between learning as a contextual activity and personal mobile technologies.

\section{Social learning}

The socio-cultural theory of learning views that learning takes place in a social context, and the forming and reforming of concepts need not necessarily take place only at the level of the individual, but that collaborative group work and sharing with peers (and others) can be a powerful way of confronting one's own conceptions, contributing to the need to restructure one's cognitive schemas [38].

Even though computers traditionally have been used as individual tools in collaborative applications, mobile devices, thanks to its mobile property, can provide the necessary support so that those collaborators can socially interact with freedom. Indeed, mobile technology can offer very important aspects to achieve a natural social interaction among collaborators [34].

\section{E. Collaborative learning}

An important issue raised in collaborative learning is the change from divergence to shared understanding and to possible construction of knowledge. The point is to understand how collaborative interactions develop over time, whether students shared knowledge building becomes richer over time, and subsequent evidence that students are able to construct their own understanding based on their interactions with others [31].

However, current interaction among students by using home computers as mediating collaboration means, demands the implicated actors to be rather statically behind a desk. Mobile technologies can remedy this situation by leading to a gain of mobility of the collaborators, which enhance their social interactions and provides the needed awareness with the learning context and location [34]. 


\section{F. Technological Perspective}

Technology is getting smaller, more personal, ubiquitous, pervasive, and powerful. Mobile devices range from the use of Personal Digital Assistants and tablet computers to context-aware devices [35]. This way, mobile technologies provide flexibility and ubiquity by accessing learning materials anytime, anywhere and adapt them to learners' personal features, preferences and interests, as well as pervasiveness by means of the latest wearable devices for learning across contexts [16].

Current college students' culture is indistinctively wireless and mobile connected. By means of a great variety of mobile and wearable devices, such as PDA, smart phones, and laptops, tablets, handheld or palmtop computers, portable media players (e.g., iPods), and so on, they are not isolate from classmates, friends and family, but incredibility flexible, fluid, communicative, and collaborative, more than ever when it comes to their social connections in their virtual life. In addition to the extremely popular social networking applications and other social tools, such as blogs, wikies, discussion boards, and so on, they are permanently connected wirelessly in any situation and for any reason. More broadly, mobile and wireless computing has altered the rhythms of social time and has changed uses of social space [2].

This technological revolution is however followed by strict accessibility, availability, security, performance that existing infrastructures can address only in small-scale and quite often not sufficiently or resulting in high costs. High performance computing, such as Grid, along with Web and $\mathrm{P} 2 \mathrm{P}$ are the enabling technology for next generation mlearning applications aiming at making affordable to meet demanding non-functional requirements appearing in this context [8]. Next section discusses further on the incorporation of Web and P2P into mobile technologies, which will be later on used to implement our Mobile Campus and thus they represent the rationale of our approach.

\section{Integration of Mobile Devices with Existing TECHNOLOGIES}

Mobile devices have many limitations such as limited computational capacity, small memory, and limited graphical user interface. They can be integrated nonetheless with different technologies including Web and P2P to support our Mobile Campus implementation, which is described in next Section.

\section{A. Integration with Web Technology}

One of the most used technologies to integrate mobile devices is the Web technology. Web application are based on centralized Client/Server architectures, where a server application provides services to Clients. Obviously, the difficulty in integrating mobile devices, that is developing mobile clients, lies in the limited capacity of mobile devices.
Additionally, the lack of standards as well as the variety of browsers make it even more complex to integrate mobile devices into existing Web applications.

In order to overcome these complexities of integrating mobile devices into Web applications, it is necessary to design and implement a module to support the specific characteristics of mobile devices. That module would offer web services accessible for mobile devices.

On the other hand, while Web clients are usually lightweight Web interfaces, in the case of mobile devices due to the lack of standards, the lightweight client interface is instead developed using Java-based technologies for mobile devices such as J2ME. The mobile clients (Midlets) developed using J2ME enables access to most available resources to support data storage, video-conferencing, etc.

\section{B. Integration with P2P Technology}

Most of nowadays online learning systems are web-based, which as centralized systems, show several limitations such as maintenance cost, scalability and having a single point of failure. $\mathrm{P} 2 \mathrm{P}$ technologies are an important alternative to develop decentralized online learning systems in which students can be more than mere clients and can use their own computational resources for task accomplishment during online learning process [4], [7], [41]. Proposals for extending P2P applications for supporting teamwork using have also been reported. For instance, in [18] the authors presented Omnix, a P2P middleware that aims to be topology independent. The middleware services it provides include support for mobile teamwork allowing different connectivity modes, support for access to various devices, and query services for distributed search and subscriptions.

Similarly as in the case of Web technology, there is no straightforward integration of mobile devices into P2P applications (see Fig. 1). Some adaptations are needed to make mobile devices peers of a standard P2P network. One of the technologies used for this purpose is the JXTA platform [5] for mobile devices. JXTA is the P2P opensource platform proposed by Sun Microsystems. It consists of a set of XML-based protocols that permit, in principle, any connected device to exchange messages and collaborate in a decentralized P2P mode with other peers in the network. JXTA supports a wide range of mobile devices such as mobile phones and PDAs. Although JXTA is said to support the integration of mobile devices into a JXTA P2P networks, it is the JXME library used to specifically support the development of P2P mobile applications. JXME library offers an architecture and a series of APIs to facilitate the access of mobile devices to $\mathrm{P} 2 \mathrm{P}$ networks by specifically addressing their limitations of computational power, memory, etc.

\section{The Mobile Campus Architecture}

For the aforementioned reasons, our Mobile Campus is based on J2ME. By using such technology to develop mobile 


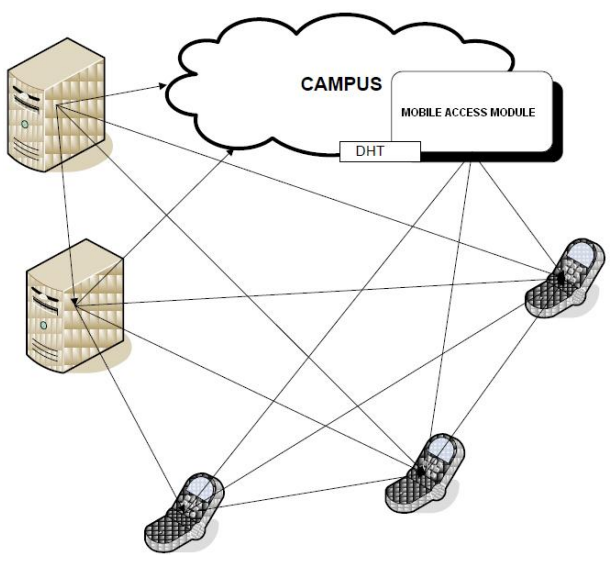

Figure 1. Integration of Mobile devices with P2P architecture.

clients to be connected to the Mobile Campus it enables both online and offline ${ }^{1}$ learning, as opposed to Web clients using browsers. One drawback, however, of using J2ME is that not all mobile devices are capable of running J2ME applications.

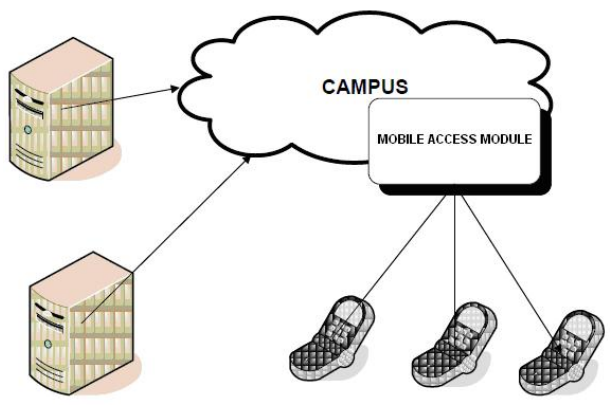

Figure 2. Integration of Mobile devices with Web architecture.

From this discussion, we can observe that actually the best option to integrate mobile devices into Web applications for our Mobile Campus is the development of a specific module, as part of Server application, that would make it possible for mobile devices to access (either via a browser or a J2ME client application) the information and services of the server application (see Fig. 2).

Another important issue in developing our Mobile Campus is the connection of mobile devices to the Campus server(s). We analyze below two most common approaches, namely proxy and proxyless architectures.

\section{A. Proxy vs. Proxyless Architectures}

Mobile devices can be connected to servers either using a proxy or a proxyless architecture.

Proxy access: Proxy is a computational device (physical or not) that acts on behalf on another computational entity. Thus, a proxy permits computational entities (network

\footnotetext{
${ }^{1}$ By storing mobile learning objects at mobile client locally.
}

nodes, peers, ...) to indirectly connect to the networks (servers, broker or rendezvous peers, ...). Clearly, the access to resources goes through communication with the proxy.

In fact, in some cases the use of proxy is the "only" solution to connect small computational devices to server applications. Using proxy architecture also enables to design richer applications for mobile and wireless devices. A special type of proxy is the so-called "transparent proxy", which combines a proxy server with a NAT in a way that the connections of client nodes to the server (via proxy) in an transparent manner (the client need not to know about the existence of the proxy).

There are several advantages of using a proxy architecture (see Fig. 3, left), briefly described next:

- Control. The workload burden is dealt with at proxy side, enabling the development of lightweight functionalities at the mobile device.

- Efficiency: Proxy can efficiently manage requests for resources by different mobile clients. Also, proxy can use responses to previous resource requests for forthcoming requests, assuming that resources are still available.

- Filtering: Proxy can filter and eventually prohibit some incoming requests from mobile clients.

There are however some disadvantages as well.

- Security and trust: Proxy could modify/falsify client's information and behavior. Moreover, proxy can see any information during communication of clients with the server, thus, security can be a concern. This is a concern in case of sensitive data, especially when such data is cached and stored at proxy side.

- Anonymity: Because mobile clients are connected through proxy, this makes difficult from the resources to distinguish their identity. This is a concern for the development applications based on trustworthiness.

- Inconsistency: Proxy could produce inconsistent states when it uses cached data (advertisements, user sessions, etc.) that might have expired.

- Network stability: Proxy could become a bottleneck for the application. In fact, if proxy goes down, the mobile clients connected to it would remain disconnected from the network.

Due to the nature of mobile devices, the proxy-based architecture is actually appropriate and efficient. The proxy can alleviate the communication burden of the mobile device with the Campus by substantially reducing the amount of data exchanged, making thus the application more efficient.

Proxyless access: Proxyless architecture (see Fig. 3, right) solves some of the proxy-base architecture, yet it is rather more difficult to integrate mobile devices in a straightforward way to server applications.

\section{B. Architecture of Mobile Campus}

In view of both architectures analyzed above and the Web approach described, the option of proxy- Web-based archi- 

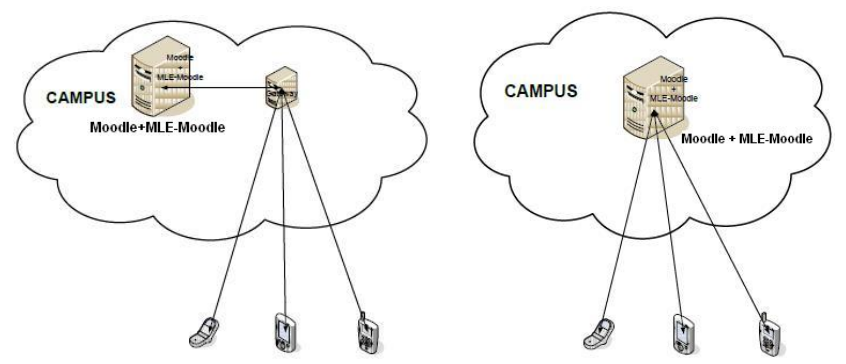

Figure 3. Proxy access (left) and Proxyless access (right).

tecture was chosen to implement the Campus application with mobile clients (see Fig. 4).

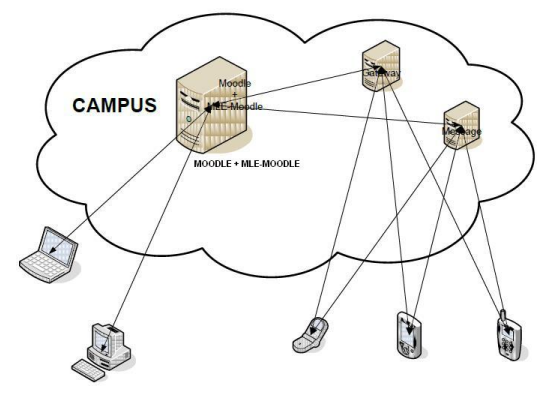

Figure 4. General view of the Mobile Campus architecture.

\section{Vi. Implementation ANd Evaluation of Mobile CAMPUS}

As a first step, we have implemented a standard Virtual Campus in Moodle. Then, the Virtual Campus is extended with MLE-Moodle module to support mobile clients according to the proxy architecture.

\section{A. Implementation on Moodle}

Moodle is an open source web-based software for developing online learning platforms following the constructivist learning paradigm (see Subsec. III-A). It can easily deployed using Apache, MySQL and PHP technologies. Moodle distinguishes for easy configuration and maintenance as well as content course creation. A great advantage of using Moodle is the easiness of content creation, including forum, questionnaires, tasks, wikis, chats, etc.

The Virtual Campus is then extended to support mobile clients. The Mobile Campus is implemented the MLEMoodle module. MLE-Moodle enables the access to the Campus of the mobile clients. Essential at this step is the adaptation of learning environment and content to be accessible and visible by mobile clients. This is achieved by using two special "servers", namely, Gateway Server, which is a proxy used by MLE to access the Campus, and, Message Server, which is a server for the instant messenger for mobile clients. Regarding the mobile client, both accessing through a browser and a Midlet Java (MLE client) have been developed.

For awareness purposes, we are currently endowing the Mobile Campus with a simpler version of awareness that consists in notifying learners of changes occurring in the virtual room (changes in objects, new messages, replied messages, etc.). In implementing the awareness features we are taking full advantage of the proxy architecture, in that, proxy does the filtering of generated events and broadcasts it to mobile clients either in the form of simple events or summaries of events.

Finally, the contents are developed as Mobile Learning Objects, which can be accessed by mobile clients. The courses content include Theory, Exercises, Discussion forum, Instant messenger, Questionnaires and Calendar (see Fig. 5).
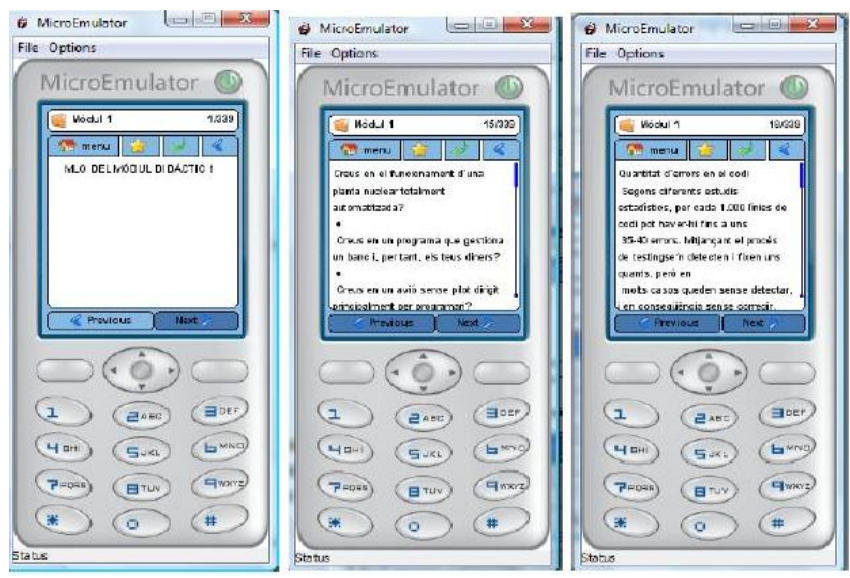

Figure 5. Example of teaching content provided by a mobile device.

\section{B. Evaluation}

The prototype has been evaluated using both mobile browser and Midlet J2EE lightweight client (MicroEmulator $^{2}$ [23] and Opera Mini Browser Simulator [29] and have been used). For the former see snapshots in Fig. 6 and for the latter see snapshots in Fig. 5.

Several tests from different perspectives have been carried out with the prototype for evaluation purposes:

- Virtual Campus: The tests were focused on setting up Moodle environment as well as the creation of courses, lessons, forums, etc.

- Mobile Campus: The test targeted to set up MLEMoodle, creation of mobile content and lessons, mobile forums, among others.

- Overall platform.: The tests targeted to check users' communications from both PC and mobile devices.

${ }^{2}$ MicroEmulator is a pure Java implementation of Java ME in Java SE. 

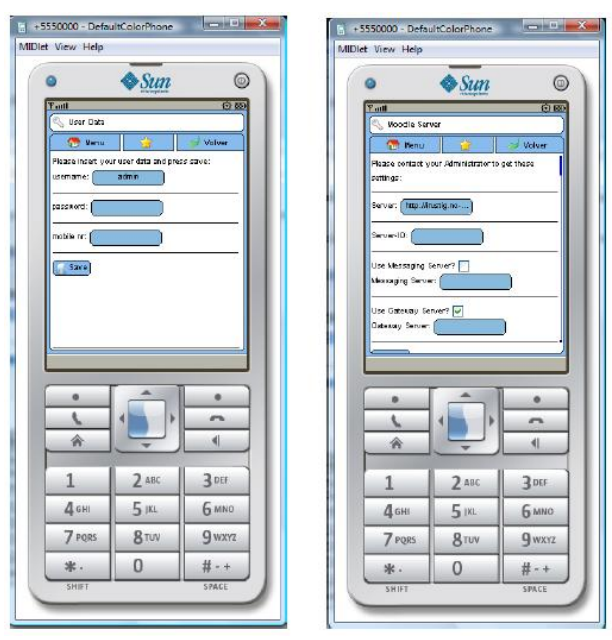

Figure 6. Login procedures for users and administrators.

For a more exhaustive evaluation of the prototype we will be using learning scenarios (as outlined in [42]) to support online learning activity.

\section{CONCLUSiOnS AND FUtURE WORK}

With the current wide spread of mobile devices and wireless technologies, e-learning is taking a new dimension, that of mobility of learners. Although mobile learning is not a new learning paradigm, it is posing pedagogical and technological challenges. Regarding the former, mobile learning should achieve the ubiquity, pervasiveness, personalization and flexibility, provided by using mobile devices any time and anywhere. Regarding the later, the lack of standards in mobile devices as well as the limited capabilities of such devices, pose difficulties in integrating mobile devices into learning environments.

In this paper we have analyzed, from both learning and technological perspectives, the development of on learning applications using mobile devices. We have considered proxy and proxyless architectures as a way to extend traditional virtual campuses with mobile clients. A prototype of a Virtual Campus is developed using MLE-Moodle -the Mobile LEarning module of Moodle- which enables mobile clients to perform online learning activities.

The results of the evaluation procedures are not conclusive due to the exploratory nature. However, it has been proved to promise significant benefits for students in the context of e-learning and education in general. We plan to keep working on our prototype with more development process and providing richer functionality to enhance and improve the mobile learning experience. In addition we plan to explore the interesting possibilities of incorporating P2P infrastructure into our approach so as to overcome certain non-functional requirements found in this context, such as full availability of the mobile teaching content, scalability, lower maintenance cost and avoiding a single point of failure, among others.

\section{ACKNOWLEDGMENT}

This work has been partially supported by the European Commission under the Collaborative Project ALICE "Adaptive Learning via Intuitive/Interactive, Collaborative and Emotional System", VII Framework Programme, Theme ICT-2009.4.2 (Technology-Enhanced Learning), Grant Agreement n. 257639. Fatos Xhafa's research work done at Birkbeck, University of London, on Leave from Technical University of Catalonia, Spain. His research is supported by General Secretariat of Universities of the Ministry of Education, Spain.

\section{REFERENCES}

[1] M. Aleksy, T. Butter, M.Schader. Architecture for the development of context-sensitive mobile applications. Mobile Information Systems 4( 2) (2008), 105-117.

[2] B. Alexander, Going Nomadic: Mobile Learning in Higher Education. Educause Review 39(5) (2004), 28-35.

[3] M. Barajas, S. Sotiriou, M. Owen, M. Lohr, Schools in Action: Pedagogical Evaluation of COLLAGE, a Case Study on Mobile and Location Game-based Learning. In Architectures for Distributed and Complex M-Learning Systems: Applying Intelligent Technologies, IGI Global, Hershey, PA, USA, (2009)

[4] K.A. Berman and F.S. Annexstein. An Educational Tool for the 21st Century: Peer-to-peer Computing. Proc. of Ohio Learning Network Conference, (2003)

[5] D. Brookshier, D. Govoni, N. Krishnan, and J.C. JXTA: Java P2P Programming. Sams Pub. (2002)

[6] Bruner, J.S. Toward a theory of instruction. Cambridge Mass: Harvard. University Press, 1996.

[7] A. Bulkowski, E. Nawarecki, and A. Duda. P2P: an Enabling Technology for Next-Generation e-learning. 4th Workshop on Online Distance Education \& E-Learning, Spain (2006).

[8] S. Caballé, F. Xhafa, L. Barolli. Using mobile devices to support online collaborative learning. Mobile Information Systems, 6(1), (2010), 27-47. IOSPress

[9] S. Caballé, F. Xhafa, Th. Daradoumis, A.A. Juan, eds. Architectures for Distributed and Complex M-Learning Systems: Applying Intelligent Technologies. In: IGI Global, Hershey, PA, USA, (2009)

[10] J. Carroll, Making Use - Scenario-Based Design of HumanComputer Interactions. The MIT Press, London, (2000)

[11] J. Carmona-Murillo, D. Corts-Polo, J.L. Gonzlez-Snchez, Designing an architecture to provide ubiquity in mobile learning. In Architectures for Distributed and Complex M-Learning Systems: Applying Intelligent Technologies, IGI Global, Hershey, PA, USA, (2009) 
[12] Chryssafidou E., Sotiriou S., Koulouris P., Stratakis M., Miliarakis A., Barajas M., Milrad M., Spikol D. (2009). Developing Tools that Support Effective Mobile and Game Based Learning: The COLLAGE Platform. Architectures for Distributed and Complex M-Learning Systems: Applying Intelligent Technologies. IGI Global: Hershey, PA

[13] EXPLOAR: http://www.ea.gr/ep/exploar

[14] C. Gutwin, S. Greenberg, M. Roseman. Workspace Awareness in Real-Time Distributed Groupware: Framework, Widgets, and Evaluation. BCS HCI 1996: 281-298.

[15] P. Hayes, D. Joyce, \& P. Pathak. Ubiquitous Learning - An Application of Mobile Technology in Education. Proceedings of World Conference on Educational Multimedia, Hypermedia and Telecommunications 2004, 1811-1816.

[16] D. Hull. Opening Minds, Opening Doors: The Rebirth of American Education. Waco, TX: Center for Occupational Research and Development, (1993)

[17] A. Kukulska-Hulme, J. Traxler, Cognitive, Ergonomic and Affective Aspects of PDA Use for Learning, in Proceedings of MLearn, (2002)

[18] R. Kurmanowytsch, E. Kirda, C. Kerer, S. Dustdar. OMNIX: A topology-independent P2P middleware, LNCS, vol. 75, (2003) 47-56.

[19] J. Lave,. Situated Learning in Communities of Practice, in: L. Resnick, J. Levine and S. Teasley ed., Perspectives on Socially Shared Cognition, American Psychological Association, Washington, DC, (1991), 63-82.

[20] R. Luckin, B. du Boulay, H. Smith, J. Underwood, G Fitzpatrick, J. Holmberg, L. Kerawalla, H. Tunley, D. Brewster and D. Pearce. Using Mobile Technology to Create Flexible Learning Contexts. Journal of Interactive Media in Education, 2005(22).

[21] F. Meawad, G. Stubbs. Supporting Mobile Access to VLE Resources through MobiGlam , Architectures for Distributed and Complex M-Learning Systems: Applying Intelligent Technologies. IGI Global: Hershey, PA, (2009)

[22] M.D. Merrill, A Task-Centered Instructional Strategy. Journal of Research on Technology in Education, 40(1), (2007) 33-50.

[23] MicroEmulator: http://www.microemu.org/

[24] D. Millard, A. Woukeu, F.B. Tao, H. Davis (2005). Experiences with writing grid clients for mobile devices. In $1 s t$ International ELeGI Conference on Advanced Technology for Enhanced Learning, BCS Electronic Workshops in Computing.

[25] MLE-Moodle: http://mle.sourceforge.net/mlemoodle/

[26] M-Learning.org: http://www.m-learning.org

[27] MLE-Engine: http://mle.sourceforge.net/mle

[28] M-Voice Learning: http://innovauoc.org/m-voicelearning

[29] Opera Mini Simulator: http://www.opera.com/mobile/
[30] D.C. Phillips \& J.C. Soltis, Perspectives on learning, Teachers College Press New York, New York, 1998.

[31] S. Puntambekar, Analyzing collaborative interactions: divergence, shared understanding and construction of knowledge. Computers \& Education. 47(3) (1996), 332-351.

[32] N.J.P. Race. Engage:enabling personalised learning across vle platforms using mobile devices. Tech. rep., Lancaster University. (2006)

[33] M. Safar, H. Sawwan, M. Taha, T. Al-Fadhli. Virtual social networks online and mobile systems. Mobile Information Systems 5(3) (2009), 233-253.

[34] M. Safar, H. Sawwan, M. Taha, T. Al-Fadhli, Virtual social networks online and mobile systems, Mobile Information Systems 5(3) (2009), 233-253.

[35] M. Sharples. Learning as conversation: Transforming education in the mobile age, Proceedings of Conference on Seeing, Understanding, Learning in the Mobile Age, (2005)

[36] M. Sharples, The design of personal mobile technologies for lifelong learning. Computers \& Education 34, (2000), 177-193.

[37] D. Spikol, and M. Milrad. Combining Physical Activities and Mobile Games to Promote Novel Learning Practices. Fifth IEEE International Conference on Wireless, Mobile, and Ubiquitous Technology in Education, (2008), 31-38.

[38] L. Vygotsky, Mind in society: The development of higher psychological processes. Cambridge: Harvard University Press, 1978

[39] C. Wattinger, D.P. Nguyen, P. Fornaro, M. Guggisberg, T. Gyalog, H. Burkhart, Problem-Based Learning Using Mobile Devices. Sixth International Conference on Advanced Learning Technologies, (2006)

[40] M. Wang, R. Shen, R. Tong, F. Yang \& P. Han. Mobile learning with cellphones and pocketpcs. Lecture Notes in Computer Science, 3583, 332-339. (2005)

[41] F. Xhafa, L. Barolli, R. Fernndez, T. Daradoumis, S. Caball. Jxta-Overlay: An interface for Efficient Peer Selection in P2P JXTA-based Systems. Computer Standards \& Interfaces, 31(5), 886-893, (2009)

[42] F. Xhafa, L. Barolli, S. Caballe and R. Fernandez. Supporting Online Scenario-based Learning with P2P Group-based Systems. Proceedings of The 13th Network-Based Information Systems (NbiS-2010), September 15-17, 2010, Japan. To appear

[43] S.J. Yang and I.Y. Chen, Providing Context Aware Learning Services to Learners with Portable Devices. In Proceedings of the Sixth IEEE International Conference on Advanced Learning Technologies, (2006)

[44] A. Zoakou, A. Tzanavari, G.A. Papadopoulos \& S. Sotiriou. A methodology for eLearning scenario development: the UNITE approach. The 6th Conference on e-Learning Copenhagen Business School, Ireland, (2007), 683-92,

[45] G. Zurita, and M. Nussbaum, Computer supported collaborative learning using wirelessly interconnected handheld computers. Computer \& Education, 42(3),(2004), 289-314, 